\title{
Prevalencia de lesiones y fitness en universitarios*
}

\author{
Carlos G. Muñoz \\ Santiago F. Reina ${ }^{* * *}$ \\ Santiago V. Cifuentes ${ }^{* * * *}$
}

Recibido: 6 de diciembre de 2020

Aceptado: 2 de febrero de 2021

Citar como:

Muñoz, C., Reina, S. y Cifuentes, S. (2021). Prevalencia de lesiones y fitness en universitarios. Revista de Investigación Cuerpo, Cultura y Movimiento, 11(2). https://doi.org/10.15332/2422474X.6758

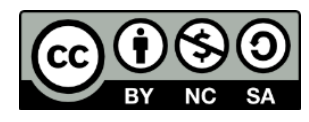

\section{Resumen}

El agrado de las personas por realizar actividad física a través de las prácticas deportivas relacionadas con las modalidades del fitness y la presencia de lesiones en sus participantes han tenido un gran aumento en las últimas décadas. Por tanto, este artículo de investigación busca evidenciar la prevalencia de lesiones en los estudiantes de la Universidad Santo Tomás (USTA) de Bogotá, Colombia. Esta investigación presenta un diseño no experimental de carácter transversal. Para el análisis se utilizó el instrumento CLUP-F con el que se identificaron la modalidad de fitness que practican, el tiempo de práctica, la cantidad de veces que

\footnotetext{
* Artículo investigativo. Investigación cuantitativa. Diseño no experimental transversal. Alcance descriptivo. Financiación propia. G.I.C.A.E.S. Universidad Santo Tomas. Bogotá. Colombia.

** Pregrado, estudiante, Universidad Santo Tomás de Aquino, Colombia. Correo electrónico: carlosgarzonm@usantotomas.edu.co; ORCID: https://orcid.org/0001-8161-3320-2081-1948

*** Pregrado, estudiante, Universidad Santo Tomás de Aquino, Colombia. Correo electrónico: foreroreinasantiago@gmail.com; ORCID: https://orcid.org/0000-0002-4876-3926

${ }^{* * * *}$ Pregrado, estudiante, Universidad Santo Tomás de Aquino, Colombia. Correo electrónico: miguelvenegas@usantotomas.edu.co; ORCID: https://orcid.org/0000-0002-3772-936X
}

Revista de Investigación Cuerpo, Cultura y Movimiento ISSN: 2248-4418 | e-ISSN: 2422-474X | DOI: https://doi.org/10.15332/2422474X 
se lesionan y la región corporal afectada. Los datos estadísticos fueron analizados utilizando hojas de cálculo en Excel (versión 2016). Los resultados indicaron que todas las modalidades presentan un índice lesivo mayor o igual al 50 \% de sus participantes, así como una predominancia de las lesiones sobre la articulación del hombro. A su vez, los practicantes de halterofilia y calistenia presentan el mayor porcentaje de participantes lesionados.

Palabras clave: fitness universitario, prácticas del fitness, prevalencia de lesiones.

\section{Prevalence of injuries and fitness in university students}

\section{Abstract}

People's liking for physical activity through sports practices related to fitness modalities and the presence of injuries in the participants have had a great increase in the last decades. Therefore, this research article seeks to demonstrate the prevalence of injuries in the students of the Universidad Santo Tomas (USTA) in Bogotá, Colombia. This research presents a non-experimental cross-sectional design. For the analysis, the CLUP-F instrument was used to identify the fitness modality practiced, the time of practice, the number of times they were injured and the body region injured. The statistical data was analyzed using Excel spreadsheets (2016 version). The result indicated that all the modalities showed an injury rate greater than or equal to $50 \%$ of their participants, as well as a predominance of injuries on the shoulder joint. In turn, calisthenics and weightlifting practitioners have the highest percentage of injured practitioners.

Keywords: university fitness, fitness practices, prevalence of injuries. 


\section{Introducción}

Todas las sociedades del mundo están pasando por un momento en el cual empiezan a tomar conciencia de la importancia en los buenos hábitos de vida como lo es la actividad física (Valencia y Bedoya, 2016). Este fenómeno social está dejando de ser algo exclusivo de fanáticos o de deportistas y está entrando por la puerta principal en los mercados y las manifestaciones culturales de los diferentes países.

Hoy en día, en cualquier continente, las personas son cada vez más activas; si observamos a Oceanía, "el 63 \% de la población australiana participa en actividades deportivas y de actividad física al menos tres veces por semana” (Australian Sport Commission, 2019, p. 19).

Del aumento de la actividad física nacen diversas prácticas enfocadas en el fitness, con las que se busca mejorar las capacidades físicas de sus practicantes empleando la innovación tecnológica y las ciencias del entrenamiento, como ocurre en las universidades de España:

[...] tienen una oferta de actividades dirigidas muy abierta, amplia y plural, en la que el objetivo de la práctica física es propiedad de la persona que realiza la actividad. Tienen tres enfoques en la oferta de actividades dirigidas, uno que se centra en la mejora de la salud y el fitness, muy condicionado por las modas [...]. (Sociedad Española de Medicina del Dolor, 2010)

En Europa, el mercado del fitness se encuentra muy consolidado y Alemania es uno de los países líderes en el uso de este tipo de entrenamientos. Es de público conocimiento como "el mercado alemán del fitness se encuentra consolidado y mantiene una posición de liderazgo en Europa con sus 10.61 millones de miembros, lo que representa casi el $18 \%$ de todos los miembros registrados en Europa" (ICEX, 2017). De igual manera, el territorio latinoamericano no se queda atrás. En México, desde 
hace tres décadas, se han consolidado las prácticas del fitness como manifestaciones culturales del ocio (Gómez et ál., 2019). Lo mismo sucede en Colombia, donde más del 50 \% de la población realiza actividad física, según encuestas realizadas (Observatorio Nacional de Salud, 2015, p. 2). También, en un estudio realizado por Mármol et ál. (2014), implementaron el entrenamiento en crossfit en niños de cuarto grado. Los investigadores pudieron demostrar la gran adherencia que tiene el niño con el desarrollo de estas prácticas y el gusto que adquieren por este tipo de entrenamientos. Todo lo anterior muestra que los ciudadanos del mundo están cada vez más involucrados con la práctica del deporte y la actividad física.

Ahora bien, el Colegio Americano de Medicina Deportiva incluye varias prácticas del fitness entre su listado anual de tendencias (Thompson, 2019). A su vez, un reporte de seguimiento de participación en deportes, fitness y recreación de Estados Unidos encontró que en las prácticas del fitness se encuentra mayor participación (Physical Activity Council, 2017). Esto demuestra la popularidad, crecimiento y adherencia que han tenido las personas respecto a las prácticas del fitness.

Así mismo, es necesario reconocer la importancia de la sistematización de los procesos de entrenamiento, así como la apropiación de la práctica del ejercicio que llevan todos los usuarios a la hora de entrenar, debido a que:

El riesgo de lesionarse realizando actividad física puede interferir con el disfrute de la participación y reducir los beneficios para la salud a largo plazo que la actividad física puede proporcionar. Las lesiones resultan ocasionando cambios negativos en las actividades diarias, el tiempo de trabajo perdido, una mala calidad de vida, discapacidad o, en casos extremos, la muerte. (Gray y Finch, 2015, p. 6)

Revista de Investigación Cuerpo, Cultura y Movimiento ISSN: 2248-4418 | e-ISSN: 2422-474X | DOI: https://doi.org/10.15332/2422474X 
$\mathrm{Al}$ reconocerse la popularidad del fitness y lo peligroso de lesionarse cuando se realiza actividad física, resulta necesario aceptar la incidencia lesiva en los practicantes de fitness. Un estudio epidemiológico logró concluir que la incidencia lesiva de esta práctica es mucho mayor respecto a otras (Finch y Gray, 2012). Otra investigación muestra cómo "aproximadamente un $15 \%$ de la población canadiense ha sufrido una lesión lo suficientemente grave como para limitar sus actividades normales" (Forbes, 2015, p. 4).

En ese orden de ideas, los entrenamientos que mayor incidencia lesiva tienen son los relacionados al fitness y sus nuevas tendencias. Una de las causas de este fenómeno lesivo se debe al desacondicionamiento físico, que ocasiona una técnica inadecuada de ejecución durante la práctica. Las lesiones causadas por la práctica de la actividad física no tienen una edad específica, pero el mayor porcentaje de lesionados se encuentra en la población de jóvenes y adultos. De hecho, en una investigación epidemiológica, se afirma que la mayoría de las personas que realizan actividad física y se lesionan están por debajo de los 35 años (Solorzano, 2017). Esto permite involucrar a la normalidad de los estudiantes universitarios de todos los países del mundo en este grupo etario y probar la importancia de reconocer la participación de los estudiantes universitarios dentro de la práctica de la actividad física.

En cuanto a las comunidades universitarias, la Pontificia Universidad Javeriana muestra cómo "los universitarios tienen un nivel de actividad física moderado-alto" (Barberán y Roa, 2016, p. 57). Así mismo, la Universidad de Pamplona, en Colombia, obtuvo como resultados de una investigación que más del $50 \%$ de la población tenía un nivel de actividad física moderado o superior (Bayona, 2018). Estudios similares realizados con universitarios chilenos mostraron que solo el " $15 \%$ de sus estudiantes no realiza actividad física" (Díaz y Castro, 2016, p. 20). A su vez, una 
investigación que trató la población universitaria de Rusia, permitió evidenciar que la totalidad de sus estudiantes realizaba algún tipo de actividad física, y más de la mitad tenían una condición física de nivel moderado o alto (Osipov et ál., 2020).

En Brasil, Martin et ál. (2008) evidenciaron que la población universitaria de este país presentó bajos niveles en los hábitos saludables, puesto que los sujetos de prueba mostraron índices de triglicéridos altos e hipertensión arterial elevada. Estos datos permiten reconocer cómo la población universitaria es físicamente activa e inactiva dependiendo de su país de origen. Estos resultados recalcan aún más la importancia de realizar un proceso de investigación relacionado con la práctica de actividad física y la población universitaria.

En Colombia, las diferentes industrias deportivas relacionadas con las prácticas del fitness también están en auge, aún más que en toda América Latina, lo cual brinda nuevas posibilidades de entrenamiento, modalidades y equipamientos que buscan una mayor intensidad (Redacción Vivir, 2014). Este hecho estimula la participación a nivel deportivo y recreativo del fitness, para la cual las divisiones de bienestar universitario de todas las instituciones de educación superior del país buscan el fomento de la actividad física, el deporte y la recreación.

La existencia de las unidades de bienestar muestra la participación de las comunidades universitarias en diversas prácticas deportivas y de actividad física, así como el crecimiento continuo de la industria del fitness en Colombia y su incremento en la realización de entrenamientos de alta intensidad.

Dicha adherencia se debe a que "en los últimos cinco años, esta industria ha conseguido posicionarse como un mercado que se mantiene en constante evolución y crecimiento, en especial, por su adaptación al 
mundo tecnológico y a la nueva era digital de las redes sociales" (Nieto y Diana, s. f., p. 4). Incluso, ha quedado en evidencia la relevancia de las investigaciones en torno a las prácticas del fitness realizadas por universitarios en Colombia.

Ahora bien, es de público conocimiento el aumento en la creación de eventos deportivos que giran en torno a las tendencias del fitness como los Crossfit Games y las competencias de modalidades afines en todo el mundo por parte de las diferentes industrias y organizaciones relacionadas con el fitness.

Las universidades no se quedan atrás. Si bien las competencias de fitness en su mayoría no hacen parte de los juegos mundiales universitarios organizados por la Federación Internacional de Deportes Universitarios (FISU), muchas de las instituciones de educación superior del mundo cuentan con estudiantes adscritos en estas modalidades de entrenamiento. Tal es el caso de la competencia de Human Fitness III, la cual apoya e incentiva la participación de eventos en municipios como Fusagasugá. Puntualmente, la Universidad Santo Tomas de Aquino (USTA) también ha venido fomentando el desarrollo y la participación en torno a las competencias del fitness. Para ello creó un torneo apoyado por la Federación Colombiana de Fisicoculturismo y Fitness, la cual es la encargada de dirigir la mayoría de las competencias en este medio.

En este orden de ideas, dado el crecimiento del fitness en el mundo, la participación a nivel nacional e internacional de los estudiantes universitarios y de sus instituciones en este tipo de eventos y la prevalencia de lesiones en quienes los practican, nace la necesidad de observar la prevalencia de lesiones en estudiantes universitarios de la USTA, para prevenir malas prácticas e identificar las modalidades de entrenamiento que resulten más lesivas para estos. Esto permite preguntarse: ¿̇cuál es la 
prevalencia de lesiones en los estudiantes practicantes de fitness pertenecientes a la Universidad Santo Tomás de Bogotá?

En ese orden de ideas, el siglo XXI ha demostrado un incremento en la cantidad de practicantes de diferentes actividades relacionadas con el ejercicio, los deportes o la actividad física, al igual que se ha visto un gran aumento en la variedad de alternativas para mantener un estilo de vida más activo y saludable.

Debido al gran auge generado por los tipos de entrenamiento de alta intensidad y del marketing usado para dar a conocer este método de entrenamiento, se han ido impactando tanto a personas físicamente activas y sedentarias casi por igual. Por esto, teniendo en cuenta que dichos entrenamientos consisten en realizar ejercicios en un umbral ventilatorio submáximo o máximo, surge la necesidad de determinar cuál es la prevalencia entre las lesiones derivadas de la práctica y los tipos de entrenamiento de alta intensidad. Por otra parte, como lo determinaron Tibana y Sousa (2018), "poco se ha informado respecto a la incidencia y prevalencia de lesiones causadas después de participar en un programa de acondicionamiento extremo" (p. 4).

Además, dentro de las investigaciones realizadas en relación con las lesiones y los entrenamientos de alta intensidad, Feito et ál. (2018) evidenciaron la existencia de lesiones en el entrenamiento, las cuales presentaron una incidencia lesiva principalmente en las articulaciones del hombro y espalda y, en una menor medida, en la rodilla, codo y muñeca. Esta medición tiene cierto parecido al estudio realizado por Toasa (2019), en el que evidenció que las articulaciones de los hombros tienen una prevalencia lesiva significativa en la zona articular del hombro. Con base en estos dos artículos se observa una correlación del entrenamiento de alta intensidad y el índice lesivo ligado principalmente a los problemas osteoarticulares y musculares. 
Estas lesiones generadas en los practicantes de fitness obligan a suspender la práctica o a limitar los movimientos y la ejecución de los ejercicios por realizar durante un determinado tiempo, lo cual puede conllevar a una baja adherencia al entrenamiento o a una lesión permanente en el participante. Esto puede afectar al entrenador, a los que trabajan con métodos de alta intensidad y a los deportistas cuyos entrenamientos se basan en el uso de estos métodos de entrenamiento.

Para este sector, las investigaciones relacionadas con la prevalencia de lesiones en el fitness de alta intensidad le permitirán realizar una mejor planeación de sus entrenamientos, en los cuales se reducirían las posibilidades de lesión para sus usuarios. Esto ayudará a generar una mayor adherencia a su entrenamiento y modo de trabajo y permitirá a las personas tener una mejor calidad de vida relacionada con el ejercicio físico para la salud o para la competencia. Además, los entrenadores que posean o no un gimnasio podrán mantener sus ingresos dentro de sus programas de entrenamiento y evitar al máximo las lesiones en sus clientes. Los practicantes del fitness se ejercitan en su mayoría de manera independiente, hecho que dificulta la cualificación de su proceso de entrenamiento, así como una retroalimentación oportuna.

En este orden de ideas, el presente proyecto se realiza con los estudiantes de la USTA, con el fin de que las oficinas de bienestar universitario, así como los encargados del gimnasio de las diferentes sedes, establezcan medidas para mitigar el riesgo de lesión relacionado con los métodos de entrenamiento o competencia en los cuales se ven involucrados los estudiantes.

También, esta investigación enriquecerá los campos de estudio en torno al ejercicio físico para la salud y la alta competencia que están relacionados con las prácticas del fitness. Por último, esta investigación podrá llevarse a cabo a una mayor escala para determinar la prevalencia de lesiones y las 
modalidades del entrenamiento de alta intensidad en una población universitaria.

De acuerdo con lo planteado anteriormente, el presente objetivo es determinar la prevalencia de lesiones en los estudiantes practicantes del fitness que pertenecen a la USTA de Bogotá, caracterizando la modalidad deportiva e identificando el tipo de lesión y la frecuencia lesiva que presentan dichos practicantes.

Tabla 1. Operacionalización de variables

\begin{tabular}{|c|c|c|}
\hline Objetivos & Variables & Indicadores \\
\hline \multirow[t]{2}{*}{$\begin{array}{l}\text { Determinar la prevalencia de lesiones en } \\
\text { los estudiantes practicantes de fitness } \\
\text { que pertenece a la USTA de Bogotá }\end{array}$} & $\begin{array}{l}\text { Prevalencia de } \\
\text { lesiones }\end{array}$ & $\begin{array}{l}\text { Lesión articular: lesión en hombro, } \\
\text { lesión en rodilla, lesión en cadera, } \\
\text { lesión en espalda, lesión en rodilla y } \\
\text { lesión en cuello de pie. Lesión por } \\
\text { movimientos repetitivos. Lesión por } \\
\text { gran impacto articular. }\end{array}$ \\
\hline & Fitness & $\begin{array}{l}\text { Fitness university, fitness sport y } \\
\text { fitness competition. }\end{array}$ \\
\hline $\begin{array}{l}\text { Caracterizar las prácticas del fitness que } \\
\text { realizan los estudiantes de la } \\
\text { universidad Santo Tomás. }\end{array}$ & $\begin{array}{l}\text { Prácticas del } \\
\text { fitness }\end{array}$ & $\begin{array}{c}\text { Técnica, intensidad RM-FC, volumen } \\
\text { y repeticiones }\end{array}$ \\
\hline
\end{tabular}

Identificar el tipo de lesiones presentadas por los practicantes de fitness que pertenecen a la USTA.
Tipos de lesiones
Musculares, articulares y tendinosas.

\footnotetext{
Evidenciar la frecuencia lesiva de las modalidades del fitness practicadas por los estudiantes de la USTA.

Modalidades del fitness

Calistenia, crossfit, entrenamiento con auto carga y halterofilia
}

Nota: definición de las variables de estudio a partir de los objetivos de investigación.

Fuente: elaboración propia.

\section{Metodología}

Se realizó una búsqueda de información en las bases de datos Science Direct, Scielo, ACSM y PubMed. Para ello, se establecieron los siguientes 
descriptores con el fin de filtrar y encontrar más rápidamente la información de calidad para la investigación: prevalencia de lesiones en el fitness, fitness en universitarios y prevalencia de lesiones en universitarios practicantes del fitness.

Del primer criterio de búsqueda de prevalencia de lesiones en el fitness, se encontró un total de 26 artículos relacionados con la investigación, distribuidos así: 9 en Scielo, 14 en ACSM y 3 en PubMed. Del criterio de búsqueda de fitness en universitarios, se encontraron 18 documentos únicamente en la base de datos Scielo. Del último criterio de búsqueda, únicamente se recuperaron 6 documentos en la base de datos Scielo. Por último, la base de datos Science Direct no cuenta con ningún documento que permita profundizar en esta investigación.

Esta búsqueda permite identificar artículos que fortalecen la investigación a nivel teórico. Por ejemplo, el estudio realizado por Klimek et ál. (2018) permitió determinar algunas de las lesiones más comunes presentadas por los practicantes de las modalidades del fitness. Además, las universidades tienen un alto gusto por promover e incentivar el fitness, y dejan ver el fenómeno lesivo que presentan algunas de las modalidades del fitness, especialmente las que son de alta intensidad. Este es el caso del crossfit, como lo describe Dominski et al. (2018), en donde existe un alta la prevalencia de lesiones en sus practicantes. En este estudio se observó que la mayoría de las lesiones se encontraron en la articulación del hombro, la espalda y rodillas.

Finalmente, la evidencia científica sobre la prevalencia de lesiones en estudiantes universitarios practicantes del fitness es poco explorada, hecho que da aún más relevancia a la investigación. 


\section{Clasificación de los tipos de lesión en el fitness}

La clasificación de las lesiones en la práctica deportiva varía según el punto de observación. Una clasificación parte del tipo de tejido lesionado. Otra, dada por Klimek et ál. (2018), plantea las clasificaciones de lesión general, lesión por uso excesivo y lesión por impacto. Desde esas perspectivas, se habla de lesiones comunes por el entrenamiento, lesiones por sobrecarga articular al ser tan repetitivos ciertos tipos de movimiento y, por último, la lesión generada por algún tipo de golpe o contacto fuerte, producida por un compañero de entrenamiento o con diversa indumentaria deportiva: pesas, bandas elásticas, barras y demás.

Otra clasificación de las lesiones sufridas por participantes de programas de fitness la aportan Gray y Finch (2014), quienes desde una perspectiva médico hospitalario clasifican las lesiones por el tipo de práctica deportiva asociada usualmente a entrenamientos aeróbicos y entrenamiento de fuerza u otros equipos.

\section{Índice de lesiones en relación con la modalidad del fitness}

Las lesiones sufridas por los participantes de fitness se encuentran muy relacionadas con la modalidad en la cual se encuentra el participante inscrito o que practica regularmente. Entre estas, se destaca el crossfit y el levantamiento olímpico o de pesas como los más estudiados en relación con las lesiones derivadas de su práctica. Así lo muestra un estudio epidemiológico que muestra cómo la estructura corporal que más se lesiona durante la práctica del crossfit es el sistema musculoesquelético, específicamente y con mayor frecuencia la columna vertebral (Hopkins et ál., 2017). Por otro lado, Klimek et ál. (2018) determinan que la articulación con un índice de lesión mayor en practicantes de la modalidad de fitness es el hombro. 
Por otro lado, la investigación de Rodrigo (2016) demostró que los practicantes de halterofilia tienden a lesionarse más el sistema osteomuscular que cualquier otro sistema del cuerpo. También, Klimek et ál. (2018) mencionan que en la práctica de la halterofilia la lesión más frecuente se da a nivel de la espalda, más exactamente la musculatura lumbar.

De acuerdo con lo anterior, se puede determinar que las lesiones en el fitness afectan principalmente el sistema musculoesquelético, puntualmente las articulaciones del hombro y la espalda baja o lumbar, cuando de halterofilia y crossfit se refieren.

\section{Principales causales de las lesiones en los participantes de diferentes programas de fitness}

Las lesiones como consecuencia del entrenamiento en fitness, al igual que en cualquier deporte o ejercicio físico, se presentan por factores múltiples como un mal calentamiento, un entrenamiento exagerado e incluso causas externas como el espacio donde se realiza la actividad.

Así las cosas, tal como lo menciona Faigenbaum (2009), una de las causales de lesión durante el entrenamiento del fitness es la exageración en la carga de entrenamiento, el sobreentrenamiento y la capacidad reducida de un individuo para tolerar dichas cargas. Esto conlleva a que el sujeto esté más predispuesto a sufrir una lesión, ya sea de tipo muscular, ósea o articular.

Otra de las causales principales de lesión en practicantes de fitness está relacionada con el nivel de experticia de los inscritos en el programa de entrenamiento. Cuando se cuenta con un nivel bajo de conocimientos sobre la técnica, se hace a una mala ejecución de los ejercicios, lo que aumenta el riesgo de sufrir algún tipo de lesión. Por otro lado, Beckham y 
Harper (2010) nos hablan de cómo la debilidad del tono y fuerza muscular, así como los rangos de movimiento reducidos, son causales de lesión. Esta situación se encuentra directamente ligada a los principiantes adscritos a los programas de fitness que los convierten en personas propensas a la lesión sin importar la modalidad del fitness que practican.

También, se encuentra la influencia del entrenador y su plan de entrenamiento sobre el sujeto que lo practica (Faingenbaum y Myer, 2012), en donde se plantean que se presenta un bajo nivel de acompañamiento e ilustración por parte de los instructores. Como consecuencia, no se genera una buena retroalimentación por parte de los entrenadores hacia los participantes, los cuales ejecutan mal el ejercicio o se sobreesfuerzan. Así mismo, en la investigación de Bianchi et ál. (2020), se encontró que hay una mayor incidencia de lesión cuando el que imparte el entrenamiento de fitness es una persona no estudiada en alguna de las ramas de las ciencias del deporte, es decir, cuando el entrenador de fitness es una persona que trabaja empíricamente las personas a su cargo.

\section{Caracterización de la lesión más común o recurrente entre los participantes de programas de fitness}

Algunas de las lesiones más comunes se presentan en el sistema osteomuscular y están muy relacionadas con el manejo de cargas externas tales como mancuernas, pesas rusas, barras, bandas de resistencia e incluso algunas máquinas presentes en los gimnasios de fitness. Las lesiones más comunes se presentan a nivel de espalda baja, muñecas, cuádriceps, manguito rotador y el hombro.

\section{El fitness en los universitarios}

El fitness ha ido adquiriendo adherencia en la población universitaria.

Tanto ha sido su acople que algunos estudios realizados han encontrado

Revista de Investigación Cuerpo, Cultura y Movimiento ISSN: 2248-4418 | e-ISSN: 2422-474X | DOI: https://doi.org/10.15332/2422474X 
que instituciones educativas han intentado hacer uso de esta modalidad en su programa de entrenamiento de educación física o cátedra de actividad física. Esto se debe a que el entrenamiento de fitness tiende a tener muchos beneficios en los sujetos que lo practican, además de su alta diversidad de modalidades de entrenamiento que existen.

Así mismo, las universidades tienen un alto gusto por promover e incentivar el fitness. Así lo estudiaron Ramos et ál. (2009), en la universidad de Caldas, donde realizaron programas de promoción de la salud y prevención de enfermedades a través de un programa de fitness, en busca de analizar los beneficios que genera esto en sus estudiantes. Estos resultaron muy significativos, ya que al estudiante le toma gran tiempo la elaboración de un entrenamiento de fitness, además del disfrute que este le genera. También implica un factor muy importante para las universidades, ya que modifica valores altos de sedentarismo además de malos hábitos de vida. Igualmente, por falta de investigaciones, no se ha podido encontrar en qué modalidades de entrenamiento del fitness los universitarios presentan una mayor adherencia o gusto.

Se ha encontrado que los estudiantes tienden a buscar la práctica del fitness no solo con el fin de salud, sino también a manera de competición, como se ha visto en los torneos realizados por la Asociación Colombiana de Universidades (Ascun) y Cerros, principales entidades en la promoción de las competiciones deportivas en las universidades. En este contexto se pueden encontrar la participación de dos modalidades del fitness: powerlifting y el weightlifting; esto ayuda a evidenciar aún más la adherencia que hay entre el universitario y la práctica del fitness.

\section{Participación de instituciones universitarias en la práctica del fitness}

Se ha mencionado que las universidades apuestan por la incorporación de las modalidades del fitness en sus programas de entrenamiento, por lo que 
han adecuado espacios para su práctica. La USTA ha adecuado espacios para la práctica de calistenia, crossfit, pilates y yoga; además, ha facilitado la práctica del fitness a través de sus modalidades de alta intensidad de HIIT, tabata, EMOM, etc. Aunque se evidencia que en algunas universidades han tenido acogida este tipo de modalidades, son pocos los estudios sobre los beneficios de estos entrenamientos, así como también las contraindicaciones que tienen el uso de estas modalidades cuando el sujeto no cuenta con la técnica necesaria para este tipo de entrenamiento. A nivel competitivo, la USTA ha buscado incentivar la competencia en las modalidades mencionadas del fitness, $\mathrm{y}$ ha brindado diferentes premiaciones. Todo esto permite ver cómo las universidades buscan incentivar el fitness.

\section{Representación o participación de atletas universitarios en las modalidades del fitness}

Al observar la cultura norteamericana, se puede evidenciar que todos los atletas de alto rendimiento de las diferentes federaciones deportivas del país deben contar con un título universitario o deben estar inscritos en alguna de estas instituciones. Esto tiene como consecuencia que haya aumento indirectamente del número de atletas universitarios en las instituciones. Para el caso de Colombia, aunque no es una norma este requisito, se puede apreciar a atletas de alto rendimiento que cuentan con un título universitario, puntualmente para las prácticas del fitness que están ligadas a la competición que se presenta en la Ascun.

Ahora bien, aunque es muy escasa la investigación sobre los atletas universitarios y la práctica del fitness, sí se puede encontrar investigaciones en lo que respecta al uso del entrenamiento del fitness en el deporte. Con estos métodos, "los resultados indican un mayor rendimiento aeróbico y anaeróbico después de dos o tres sesiones de HIIT 
por semana durante un período de cinco a diez semanas, además del entrenamiento normal" (Engel y Sperlich, 2014, p. 4). Esto evidencia la importancia de investigar los beneficios que genera estas modalidades en los atletas universitarios, ya que va a generar un mayor acople y gusto hacia su ejecución, sin contar los beneficios que otorga hacia la salud del deportista.

\section{Prevalencia de lesiones en los universitarios practicantes de fitness}

En el transcurso de los años, la comunidad universitaria ha incrementado la participación sobre las actividades culturales que giran en torno a la práctica de la actividad física. Estos procesos son posibles gracias a la gestión de las universidades dentro de sus instalaciones y sus allegados; a través de la aceptación de estudiantes deportistas, de la adecuación de recursos y escenarios, y de la cualificación de instructores y docentes que enriquezcan estas prácticas. Así las cosas, la gestión de las diversas instituciones universitarias permite visibilizar, masificar y enriquecer la práctica deportiva de sus integrantes, cuidando al máximo su integridad. Con todas estas facilidades que se les brinda a los estudiantes universitarios se esperaría tener una vivencia bastante enriquecedora en torno a la práctica deportiva, con lo cual se aportar a una comunidad físicamente más activa y, por qué no, cultivar a deportistas de alto rendimiento que participen de las justas deportivas universitarias.

$\mathrm{Al}$ observar cuáles son las organizaciones principales que rigen el deporte universitario en el mundo, se encuentra en primera instancia a la Federación Internacional de Deportes Universitarios (FISU), que cuenta con dos modalidades de competencia en sus juegos mundiales asociadas al fitness desde el campo de la halterofilia: powerlifting y weightlifting.

Así las cosas, este panorama permite ver el interés del sector universitario y deportivo en impulsar el deporte que los represente. Pero este 
incremento en la participación es bastante beneficioso, aunque preocupante, debido al riesgo inminente de padecer una lesión durante la práctica deportiva. Es de conocimiento público que se evidencian lesiones durante las distintas prácticas o competencias de las diferentes disciplinas deportivas existentes, puntualmente del fitness.

Indagar las sesiones en las competencias deportivas universitarias resulta bastante amplio y relevante, debido al posicionamiento sociocultural de estas en el mundo. Pero es más urgente revisar las lesiones que padecen los practicantes de fitness, más allá de los competidores, no solo por la cantidad de practicantes de deporte universitario, que es mayor a la cantidad de deportistas universitarios de alto nivel, sino también porque, si bien es una mayor población, estos practicantes del fitness universitario son las bases y reservas deportivas que terminan por ser vinculadas a este tipo de programas.

Adicional a lo anterior, la revisión bibliométrica así como la inspección sobre bases de datos y las revistas electrónicas afines que se estudiaron para esta investigación no arrojaron ningún registro epidemiológico o informativo sobre las predominancias en la presencia de lesiones osteomusculares de una universidad en comparación con otra. Ya fuera durante la competencia o durante la práctica deportiva, debido al poco registro que hay de estos sucesos durante los eventos.

Respecto al marco metodológico, este estudio tiene un enfoque cuantitativo y un alcance experimental. El diseño no experimental de carácter transversal se realiza con una muestra no representativa de 21 estudiantes de la USTA. Su muestreo es probabilístico debido a que cualquier unidad de análisis de la muestra contará con la misma probabilidad de ser seleccionada dentro de la investigación. A su vez, la muestra está estratificada, ya que se subdividió la población en grupos de interés. Se utilizará también una técnica estratificada uniforme, ya que se 
busca tener la misma cantidad de participantes en cada uno de los grupos, y la técnica de análisis por distribución de frecuencias.

La herramienta usada para la recolección de información es una encuesta, la cual se desarrolla por medio de la plataforma Drive usando la herramienta de formularios de Google. Esta encuesta se diseña con el objetivo de tener una mayor precisión en los resultados teniendo en cuenta que los instrumentos disponibles limitaban la investigación e ignoraban variables pertinentes a este ejercicio investigativo. Los criterios para seleccionar la muestra fueron:

Criterios de inclusión

Estudiantes actualmente inscritos en la USTA.

Estudiantes de la universidad que practican una modalidad deportiva relacionada al fitness.

Criterios de exclusión

Diligenciamiento inadecuado del formulario.

Practicantes de modalidades deportivas no afines a la investigación.

Por otro lado, la encuesta denominada "Cuestionario de lesiones para practicantes universitarios de fitness" (CLUP-F) consta de dos secciones: en la primera se expresa el consentimiento informado, el cual debe ser aceptado por el participante para poder proseguir a la siguiente sección. La segunda sección se encuentran un total de 20 preguntas, siendo las primeras 5 preguntas de caracterización sociodemográfica. Las preguntas restantes están directamente relacionadas con la investigación y hacen referencia a modalidades de fitness practicadas, experiencia, lesiones sufridas, zona lesionada y demás. Finalmente, cabe aclarar que este cuestionario se realiza teniendo en cuenta las variables determinadas para investigar en el inicio de la investigación. El formulario contará con una 
validez de contenido y con una confiabilidad aceptable de 0.7 en el alfa de Cronbach.

El diligenciamiento del formulario CLUP-F inicia el día 19 de octubre de 2020 y se extiende por una semana, hasta el día 26 de octubre de 2020. Esta aplicación y recolección de información se realiza únicamente por medios virtuales debido a las medidas impuestas a causa del COVID-19 y en búsqueda de proteger tanto a los investigadores como a los encuestados.

Por esta razón, el enlace de acceso al formulario es enviado por WhatsApp a los encuestados. Se les hace envío y solicitud de diligenciamiento de la encuesta, así como la petición de compartir la misma para así lograr una mayor cobertura de la investigación. También se envió por el correo institucional de los investigadores. Terminada la semana de plazo para la recolección de información, se verifican las respuestas obtenidas y se procede al análisis de los resultados.

Por último, se plantea la hipótesis para resolver dentro de la investigación:

\section{Hipótesis}

Hi: Los practicantes de fitness no tienen una alta prevalencia de lesiones. Ho: Los practicantes de fitness tienen una alta prevalencia de lesiones. Ha: Solo los practicantes de una o varias prácticas del fitness tienden a tener una alta prevalencia de lesiones. 


\section{Resultados}

Tabla 2. Características de la práctica y de su entrenamiento

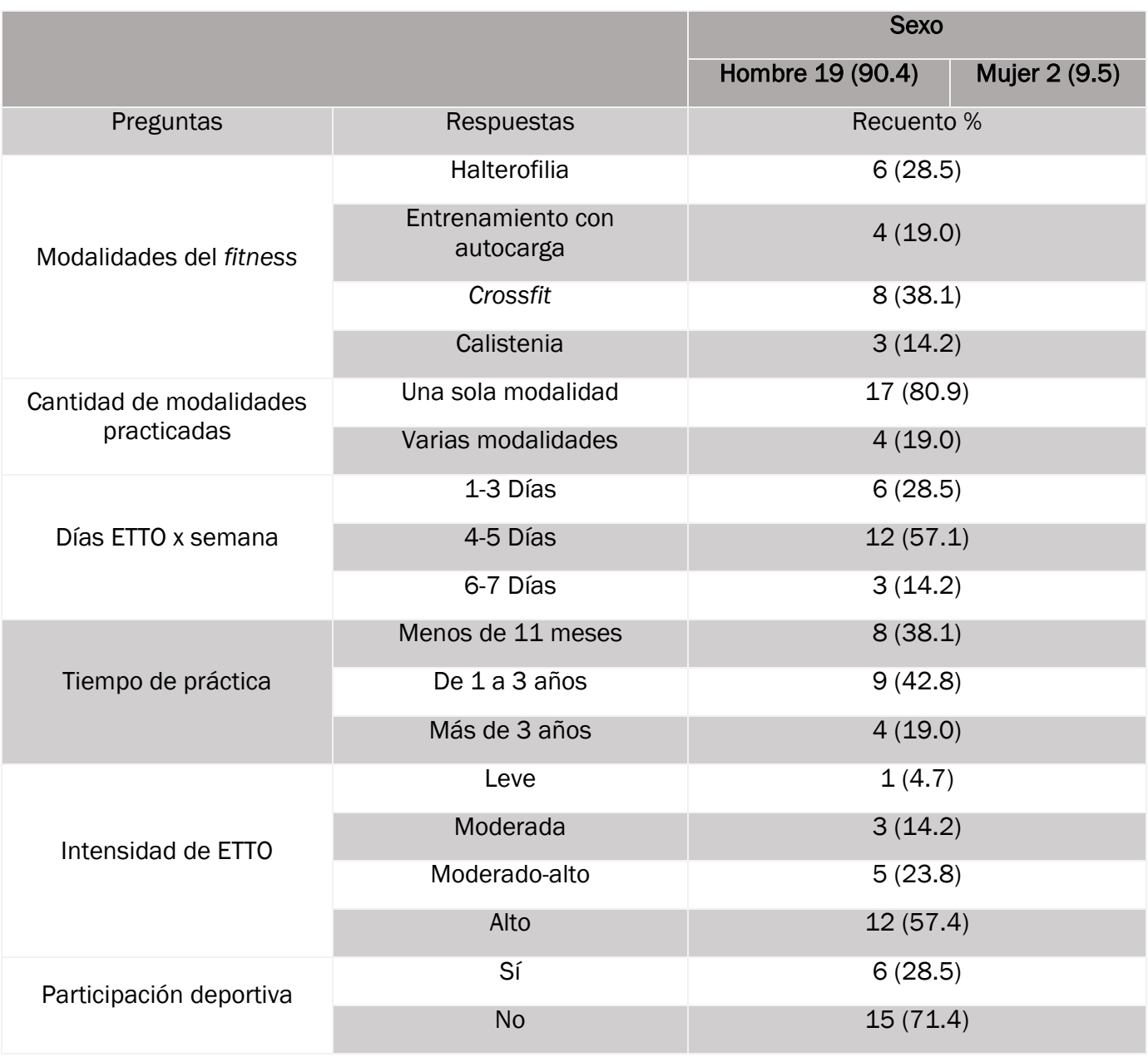

Nota: nivel de participación de los sujetos en las modalidades del fitness y su intensidad de participación.

Fuente: elaboración propia.

La tabla 2 muestra las características de los participantes respecto a las modalidades de fitness que practican. La mayoría de la muestra corresponde al sexo masculino con un 90.4\%. De los participantes encuestados la mayoría practican solo una modalidad deportiva, en su mayoría, crossfit. Los encuestados tienen una intensidad alta de 
entrenamiento y entrenan más de 4 días a la semana. Sin embargo, solo el $28.5 \%$ de la muestra ha participado en competencia afines.

Figura 1. Distribución por recuento y porcentaje de la región muscular afectada según la práctica del fitness.

Comparación de la región corporal lesionada y la modalidad de fitness

$100,00 \%$

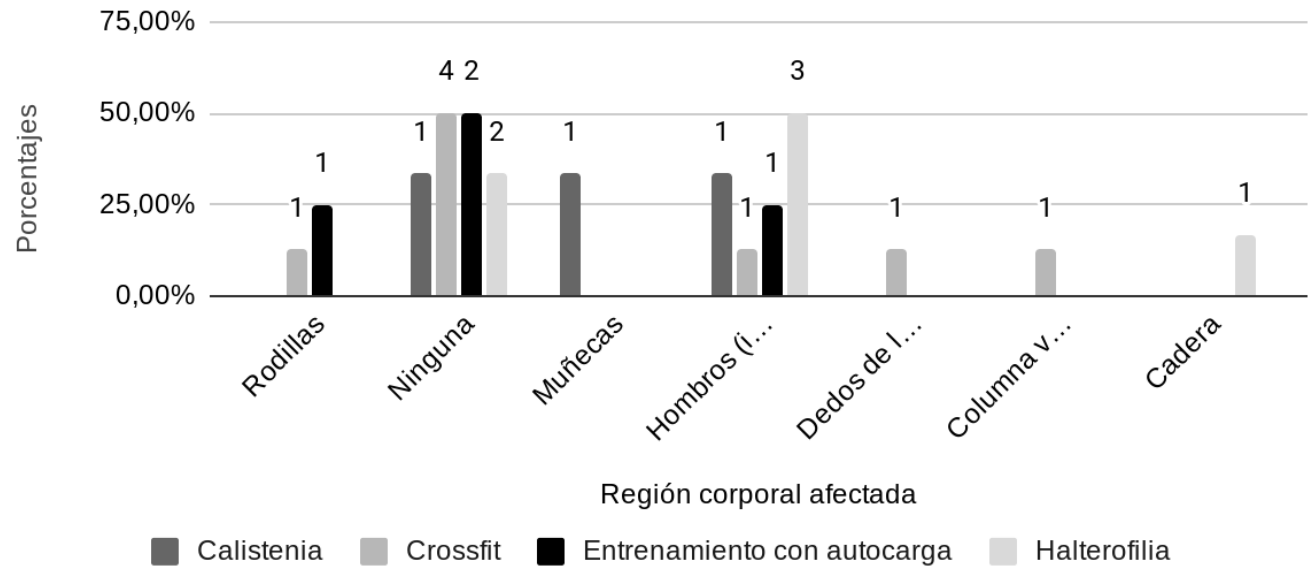

Nota: los números de las barras representan la cantidad de participantes por modalidad.

Fuente: elaboración propia.

La figura 1 ofrece una distribución del segmento corporal lesionado en relación con las modalidades del fitness practicadas. De estas últimas la que presenta mayor cantidad de población lesionada son las de halterofilia y crossfit. Por otro lado, la calistenia presenta el $66.6 \%$ de sus practicantes encuestados lesionados. Adicional a esto, el entrenamiento con autocarga presenta el $50 \%$ de sus participantes lesionados. Finalmente, la articulación del hombro es el segmento corporal que presenta un índice lesivo mayor que los otros segmentos, independientemente de la modalidad deportiva. 
Tabla 3. Características relacionadas con las lesiones

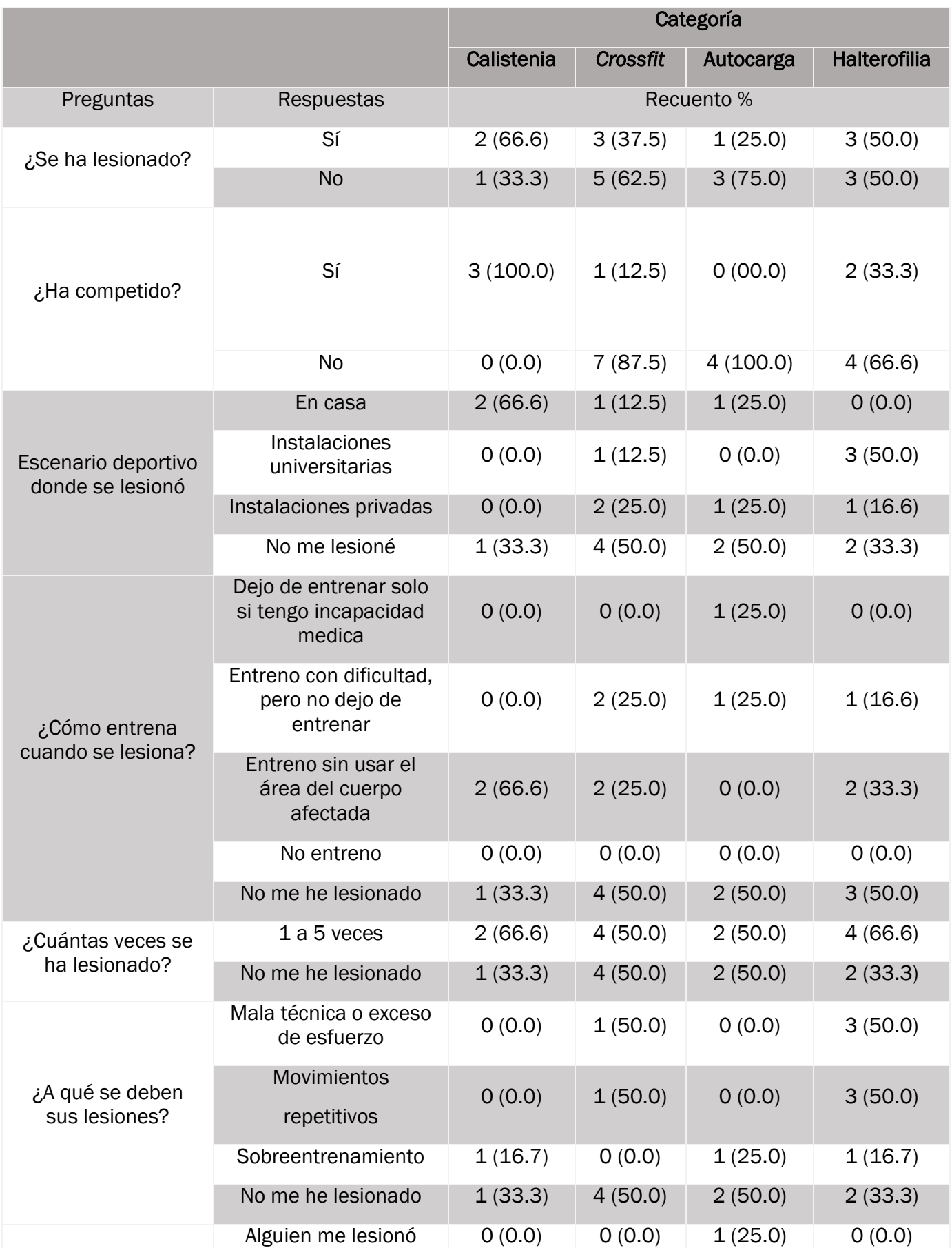

Nota: busca evidenciar el índice lesivo existente entre los practicantes de

fitness y el impacto articular que se presenta en estas modalidades.

Fuente: elaboración propia.

Revista de Investigación Cuerpo, Cultura y Movimiento ISSN: 2248-4418 | e-ISSN: 2422-474X | DOI: https://doi.org/10.15332/2422474X

Vol. 11 N. ${ }^{\circ} 2$ | julio-diciembre de 2021 
La tabla 3 muestra las características de los practicantes del fitness en relación con las lesiones. Se puede apreciar que todas las modalidades presentan practicantes lesionados. Los escenarios deportivos donde se presentan una mayor incidencia de lesiones son en casa y en instalaciones privadas. De los practicantes lesionados, la mayoría entrenan sin usar el área del cuerpo afectada. A su vez, los practicantes lesionados presentan un máximo de 5 lesiones desde el inicio de su práctica deportiva. Las lesiones en su mayoría son causadas por una mala técnica o esfuerzo excesivo, así como por movimientos repetitivos.

En ese orden de ideas, los resultados arrojados por esta investigación permitieron discriminar las lesiones en relación con el método de entrenamiento usado por cada uno de los encuestados. Dentro de estos se encontró el entrenamiento de autocarga, halterofilia, crossfit y calistenia. Principalmente el crossfit resulta ser dentro de la investigación la modalidad del fitness más practicada por los estudiantes de la USTA. Por otra parte, la modalidad de calistenia fue la que mayor índice lesivo presentó en comparación con las otras modalidades anteriormente mencionadas. También se realizó una correlación entre la región corporal afectada durante la lesión, la cual evidenció que la región más afectada de la modalidad de halterofilia es la articulación del hombro, seguido por la cadera.

Por otro lado, en la modalidad de entrenamiento de autocarga se evidencia que las lesiones de sus participantes son en el hombro y rodilla. Este hallazgo contrasta con la investigación realizada por Klimek et al. (2018), quienes determinan que los practicantes del fitness en cualquiera de sus modalidades tienden a lesionarse principalmente el hombro y estas lesiones son usualmente de carácter músculo esquelético.

En cambio, en la modalidad del crossfit las lesiones más recurrentes se presentan habitualmente en el esqueleto axial. Esto se asemeja a los 
resultados de la investigación de Hopkins et ál. (2017), que determinan que la región más afectada en cuestión de lesiones en los practicantes de esta modalidad es la columna vertebral y que esta lesión es principalmente de carácter musculoesquelético.

Por último, los resultados de esta investigación comprueban la hipótesis nula, pues muestra que todas las modalidades de fitness practicadas presentan el $50 \%$ o más de su población estudiantil lesionada.

\section{Conclusiones}

Se evidenció una alta prevalencia lesiva en todas las modalidades practicadas y en mayor medida en calistenia y autocarga. La modalidad del fitness más practicada en la universidad es el crossfit, la cual cuenta con el $50 \%$ de sus practicantes lesionados en diferentes zonas articulares, sobre todo, en la articulación del hombro, que es la que más se tiende a lesionar independientemente de la modalidad que se practique. Además de esto, los escenarios deportivos que se prestan para una mayor exposición a una lesión son en casa y en instalaciones privadas; así, se salvaguarda la asesoría de los entrenadores de los gimnasios dentro de las instalaciones universitarias procurando cualificar los procesos de entrenamiento en las instalaciones previamente mencionadas.

Por otro lado, es importante reconocer que las investigaciones relacionadas con la incidencia y prevalencia de lesiones en practicantes universitarios del fitness deben abordarse con mayor profundidad. Esto permite orientar a los entrenadores hacia la cualificación de la enseñanza sobre estas prácticas, así como brindarle herramientas para la prevención de los fenómenos lesivos en sus practicantes. 


\section{Referencias}

Sociedad Española de Medicina del Deporte. (2010). Plan integral para la actividad física y el deporte. http://femede.es/documentos/Universidadv1.pdf

Australian Sport Commission. (2019). Annual Report 2018-2019. https://www.sportaus.gov.au/ data/assets/pdf file/0007/716119/ASC-AnnualReport-20182019.pdf

Barberán, J. D. y Roa, J. D. (2016). Actividad física en universitarios y su relación con autoeficacia y percepción de riesgo [tesis de pregrado]. https://doi.org/10.1174/021037008783487129

Bayona, J. (2018). Niveles de sedentarismo en estudiantes universitarios de pregrado en Colombia. Revista Cubana de Salud Pública. http://www.revsaludpublica.sld.cu/index.php/spu/article/view/881/1135

Beckham, S. y Harper, M. (2010). Functional training: Fad or here to stay? ACSM's health y Fitness Journal, 14(6) 24-30. https://doi.org/10.1249/fit.obo13e3181f8b3b7

Bianchi, F., Labbate, M., Castellana, M., Stefanizzi, P., De Nitto, S., Notarnicola, A. y Tafuri, S. (2020). Epidemiology of injuries among amateur athletes who attended fitness activities: the role of the qualification of the trainer. The Journal Of Sports Medicine And Physical Fitness, 6o(3), 422-7. https://doi.org/10.23736/s00224707.19.10068-0

Faigenbaum, A. D. y Myer, G. D. (2012). Exercise deficit disorder in youth: play now or pay later. Current sports medicine reports, 11(4), 196-200.

https://doi.org/10.1249/JSR.obo13e31825da961

Diaz, J. y Castro, M. (2016). Desempeño motor y hábitos de actividad física en estudiantes universitarios en Costa Rica. MHSalud, 2(12) 15-24.

https://doi.org/10.15359/mhs.12-2.2

Dominski, F. H., Siqueira, T. C., Serafim, T. T., y Andrade, A. (2018). Perfil de lesões em praticantes de CrossFit: revisão sistemática. Fisioterapia e Pesquisa, 25(2), 229239. https://doi.org/10.1590/1809-2950/17014825022018

Engel, F. A., y Sperlich, B. (2014). High-intensity interval training for young athletes. Wiener medizinische Wochenschrift, 164(11-12), 228-238. https://doi.org/10.1007/s10354-014-0277-X 
Faigenbaum, A. (2009). Overtraining in young athletes: How much is too much?. ACSM's health y Fitness Journal, 4(13), 8-13. https://doi.org/10.1249/fit.obo13e3181aaeoao

Feito, Y., Burrows, E. K., y Tabb, L. P. (2018). A 4-year analysis of the incidence of injuries among CrossFit-trained participants. Orthopaedic journal of sports medicine, 6(10), 1-8. https://doi.org/10.1177/2325967118803100

Finch, C. y Gray, S. (2012). The epidemiology of hospital-treated for injuries sustained in the health and fitness sector. Journal of Science and Medicine in Sport, 1(15), 1-18. https://doi.org/10.1016/j.jsams.2012.11.265

Forbes, S. (2015). Injury prevention in sport and physical activity in Ontario: What's the game plan? Toronto: ON.

http://www.playsafeinitiative.ca/uploads/3/o/6/4/3064403/play safe white pa per 2016.pdf

Gómez, L. Cervantes, J. y Marqués, A. (2019). Desarrollo y actualidad del mercado fitness en Puerto Vallarta, México. Revista digital de educación física EmásF, 6o(1), 8697. https://dialnet.unirioja.es/descarga/articulo/7133739.pdf

Gray, S. y Finch, C. (2014). Epidemiology of Hospital-Treated Injuries Sustained by Fitness Participants. Research Quarterly for Exercise And Sport, 86(1), 81-87. https://doi.org/10.1080/02701367.2014.975177

Gray, S. y Finch, C. (2015). The causes of injuries sustained at fitness facilities presenting to Victorian emergency departments-identifying the main culprits. Injury Epidemiology Journal. https://doi.org/10.1186/s40621-015-0037-4

Hopkins, B., Cloney, M., Kesavabhotla, K., Yamaguchi, J., Smith, Z., Koski, T., Hsu, W. y Dahdaleh, N. (2017). Impact of CrossFit-Related Spinal Injuries. Clinical Journal of Sport Medicine, 29(6), 482-485.

https://DOI.org/10.1097/ism.0000000000000553

Klimek, C., Ashbeck, C., Brook, A. y Durall, C. (2018). Are Injuries More Common With CrossFit Training Than Other Forms of Exercise? Journal Of Sport Rehabilitation, 27(3), 295-299. https://doi.org/10.1123/jsr.2016-0040

Revista de Investigación Cuerpo, Cultura y Movimiento ISSN: 2248-4418 | e-ISSN: 2422-474X | DOI: https://doi.org/10.15332/2422474X 
España Exportación e Inversiones. (2017). La industria del fitness en Alemania [en línea].

https://www.icex.es/icex/GetDocumento?dDocName=DOC2019819239yurlNoAcc eso=/icex/es/registro/iniciarsesion/index.html?urlDestino=https://www.icex.es:443/icex/es/navegacionprincipal/todos-nuestros-servicios/informacion-de-mercados/estudios-demercados-y-otros-documentos-de-comercioexterior/DOC2019819239.htmlysite=icexES

Mármol, A. G., Martínez, B. J. S. A., Llamazares, M. P. y Marín, A. R. (2014). El crossfit en la educación física escolar. EmásF: revista digital de educación física, 30, 19-34. https://doi.org/10.28997/ruefd.voi13.5

Martins, F., Chagas, M., Muniz, D. y Oliveira, L. (2008). Estado nutricional, medidas antropométricas, nivel socioeconómico y actividad física en universitarios brasileños. Nutrición Hospitalaria, 23(3), 234-241. https://scielo.isciii.es/pdf/nh/v23n3/original6.pdf

Nieto, D. P. (s. f.). Análisis de la incidencia fitness en la sociedad femenina actual. https://repository.usta.edu.co/handle/11634/12470?show=full

Observatorio Nacional de Salud. (2015). Actividad Física en Colombia. https://www.ins.gov.co/Direcciones/ONS/Boletines/boletin5-AF/indexboletin/Boletin5ONS.pdf

Osipov, A., Potop, V., Nagovitsyn, R., Zemba ,E., Knyazev, A., Orlova, I., Ratmanskaya, T. y Iermakov, S. (2020). Indicators of physical activity and fitness of male students at Russian universities. Physical education of students. https://doi.org/10.15561/20755279.2020.0105

Physical Activity Council. (2017). The Physical Activity Council's annual study tracking sports, fitness, and recreation participation in the US. http://s3.amazonaws.com/ustaassets/assets/822/15/papc study.pdf

Ramos, S., Alzate, D., Ayala, E., Franco, M. y Sánchez, A. (2009). Fitness profile of universidad de caldas students. Hacia la Promoción de la Salud, 14(1), 23-34. https://www.researchgate.net/publication/262457319 FITNESS PROFILE OF UNIVERSIDAD DE CALDAS STUDENTS/references

Redacción vivir. (2014). La industria del FITNESS pisa fuerte. El Espectador. https://www.elespectador.com/noticias/salud/la-industria-fitness-pisa-fuerte/

Revista de Investigación Cuerpo, Cultura y Movimiento ISSN: 2248-4418 | e-ISSN: 2422-474X | DOI: https://doi.org/10.15332/2422474X Vol. 11 N.o 2 | julio-diciembre de 2021 
Rodrigo, J, B. (2016). ¿Es crossfit un deporte de alto riesgo? Estudio ergonómico de la postura corporal de las personas que realizan crossfit en la ciudad de Quito [tesis de pregrado]. Repositorio Digital USFQ.

https://doi.org/10.26820/reciamuc/1.4.2017.649-683

Solorzano. (2007). Ejercicios físicos y prevalencia de lesiones osteomusculares en adultos jóvenes del instituto superior de educación policial de la escuela de comando y estado mayor. (Tesis de maestría). https://doi.org/10.14482/esal.2.10151

Thompson, W. (2019). Worldwide survey of fitness trends for 2020. ACSM's Fitness Journal, 23(6) 10-18. https://doi.org/10.1249/FIT.0000000000000526

Tibana, R. A. y De Sousa, N. M. F. (2018). Are extreme conditioning programmes effective and safe? A narrative review of high-intensity functional training methods research paradigms and findings. BMJ open sport y exercise medicine, 4(1).

https://doi.org/10.1136/bmjsem-2018-000435

Toasa, K. M. (2019). Prevalencia de lesiones músculo esqueléticas y su causa en deportistas de alto rendimiento (Crossfit) de la ciudad de Quito, en el periodo 2019. Bachelor's thesis, PUCE-Quito, 1-61. https://doi.org/10.26820/reciamuc/1.4.2017.649-683

Valencia, C. F. y Bedoya, D. E. (2016). Tendencias del estilo de vida fitness en Colombia. (Tesis de pregrado). Recuperada de la base de datos de la universidad ICESI. https://doi.org/10.21840/siic/156335 